\title{
Clinical characteristics and outcome of lgG4-related disease with hypocomplementemia: a prospective cohort study
}

Linyi Peng ${ }^{\dagger}$, Hui Lu ${ }^{\dagger}$, Jiaxin Zhou, Panpan Zhang, Jiegiong Li, Zheng Liu, Di Wu, Shangzhu Zhang, Yunjiao Yang, Wei Bai, Li Wang, Yunyun Fei*, Wen Zhang ${ }^{*}$ (D, Yan Zhao, Xiaofeng Zeng and Fengchun Zhang

\begin{abstract}
Background: Immunoglobulin G4-related disease (IgG4-RD) is a newly recognized systemic, immune-mediated, and fibro-inflammatory disease. Hypocomplementemia was found in part of IgG4-RD patients especially in the setting of active disease.

Objectives: This study aimed to clarify the clinical features, treatment efficacy, and outcome in IgG4-RD patients with hypocomplementemia.

Methods: 312 lgG4-RD patients were recruited in our prospective cohort conducted in Peking Union Medical College Hospital. Patients were divided into hypocomplementemia group and normal complement group according to serum C3 and C4 levels measured at baseline before treatment. Low serum C3 levels $(<0.73 \mathrm{~g} / \mathrm{L})$ and/ or C4 levels $(<0.10 \mathrm{~g} / \mathrm{L})$ were defined as hypocomplementemia. Demographic data, clinical characteristics, laboratory parameters, treatment, and outcome of two groups were analyzed and compared.

Results: Hypocomplementemia was identified in 65 (20.8\%) cases of untreated IgG4-RD patients at baseline. The average age of hypocomplementemia group was $55.85 \pm 10.89$ years, with male predominance (72.3\%). Compared with normal complement group, patients with hypocomplementemia were likely to have more involved organs, higher lgG4-RD responder index (IgG4-RD Rl), and higher laboratory parameters such as counts of eosinophils, inflammatory markers, immunoglobulin G (lgG), IgG1, lgG3, IgG4, and IgE. In addition, lymph nodes, lacrimal gland, submandibular gland, parotid gland, paranasal sinus, bile ducts, and prostate gland were more commonly affected $(p<0.05)$. Serum C3 and C4 showed a significant positively correlation with each other. Both C3 and C4 were negatively correlated with the number of involved organs, $\operatorname{lgG}, \operatorname{lgG} 3$, $\operatorname{lgG} 4$, and $\operatorname{lgG} 4-\mathrm{RD} R \mathrm{Rl}$, as well as positively (Continued on next page)
\end{abstract}

\footnotetext{
* Correspondence: feiyunyun2013@hotmail.com; zhangwen91@sina.com

'Linyi Peng and Hui Lu contributed equally to this work.

Department of Rheumatology and Clinical Immunology, Chinese Academy of Medical Sciences \& Peking Union Medical College, National Clinical Research Center for Dermatologic and Immunologic Diseases (NCRC-DID), Ministry of Science \& Technology; State Key Laboratory of Complex Severe and Rare Diseases, Peking Union Medical College Hospital (PUMCH); Key Laboratory of Rheumatology and Clinical Immunology, Ministry of Education, No.1 Shuai Fu Yuan, Dong Cheng District, Beijing 100730, China
}

(c) The Author(s). 2021 Open Access This article is licensed under a Creative Commons Attribution 4.0 International License, which permits use, sharing, adaptation, distribution and reproduction in any medium or format, as long as you give appropriate credit to the original author(s) and the source, provide a link to the Creative Commons licence, and indicate if changes were made. The images or other third party material in this article are included in the article's Creative Commons licence, unless indicated otherwise in a credit line to the material. If material is not included in the article's Creative Commons licence and your intended use is not permitted by statutory regulation or exceeds the permitted use, you will need to obtain permission directly from the copyright holder. To view a copy of this licence, visit http://creativecommons.org/licenses/by/4.0/ The Creative Commons Public Domain Dedication waiver (http://creativecommons.org/publicdomain/zero/1.0/) applies to the data made available in this article, unless otherwise stated in a credit line to the data. 
(Continued from previous page)

correlated with IgA and hypersensitive C reactive protein (hsCRP). 64 (98.5\%) patients responded quickly to initial therapy at a 3-month follow-up. Fifteen (23.1\%) patients relapsed during follow-up with mean recurrence time of $14.2 \pm 13.8$ months. Compared with normal complement group, there was no significant difference of relapse rate in two groups $(P=0.401)$.

Conclusions: Clinical characteristics of IgG4-related disease with hypocomplementemia differ from normal complement group. Serum C3 and C4 at baseline before treatment could be biological markers for disease activity. IgG4-RD with hypocomplementemia responded well to treatment and had no significant difference of relapse rate in IgG4-RD with normal complement.

Keywords: IgG4-related disease, Hypocomplementemia, Complement, Immunoglobulin G4, Relapse

\section{Introduction}

Immunoglobulin G4-related disease (IgG4-RD) is a newly recognized multi-organ, immune-mediated, and fibro-inflammatory disease with pathologically characterized by IgG4-positive lymphoplasmacytic infiltration, storiform-fibrosis, and obliterative phlebitis. IgG4-RD affects nearly every organ, particularly the lacrimal glands, salivary glands, pancreas, bile ducts, lungs, kidneys, retroperitoneum, artery, thyroid gland, meninges, and orbits. Approximately, a quarter to a third of patients with active IgG4-RD have hypocomplementemia defined by the low level of complement component $\mathrm{C} 3$ or $\mathrm{C} 4[1,2]$.

Complement is one of the first lines of defense against infections by promoting inflammation and orchestrating opsonization of pathological material, and other critical roles including disposal of immune complexes and apoptotic cellular debris [3]. In addition, it serves as a functional bridge between the innate and adaptive immune systems by enhancing antibody responses and regulating $\mathrm{B}$ and $\mathrm{T}$ cells activation, playing important roles in the development of numerous inflammatory diseases [3-5]. There are three complement pathways: classical, alternative, and Mannan-binding lectin (MBL) pathways. The cleavage of $\mathrm{C} 3$ and $\mathrm{C} 5$ leads to the production of the membrane attack complex [5].

Excessive and uncontrolled activation of the complement has been implicated in a series of autoimmune diseases with different pathway and mechanism such as systemic lupus erythematosus (SLE), antiphospholipid syndrome (APS), and anti-neutrophil cytoplasmic antibody-associated vasculitis (AAV). In SLE, the predominant role of the classical pathway in initiation of complement activation, while alternative pathway amplification loop caused complement-mediated damage [4, $6,7]$. In AAV, the alternative pathway and C5a in particular acted as a bridge that links the inflammation and coagulation process [8]. As so far, the role of complement in the pathogenesis of IgG4-RD and which was the activation pathway had not been clarified.

In this study, we focus on clinical features, serum markers, treatment response, and outcome in IgG4-RD with hypocomplementemia; meanwhile, we are trying to investigate whether complement $\mathrm{C} 3$ and $\mathrm{C} 4$ levels at disease onset could be a biological marker for disease activity and prognosis.

\section{Methods \\ Patient enrollment}

In our prospective cohort of IgG4-RD carried out in the Peking Union Medical College Hospital (registered as ClinicalTrials.gov ID: NCT01670695), 312 newly diagnosed patients were enrolled from January 2014 to January 2019, who fulfilled the 2011 comprehensive diagnostic criteria $[9,10]$, had complement tested at baseline, and had been followed up for more than 6 months. Patients with low serum C3 levels $(<0.73 \mathrm{~g} / \mathrm{L})$ and/or C4 levels $(<0.10 \mathrm{~g} / \mathrm{L})$ before treatment were defined as hypocomplementemia group. The diagnosis of IgG4-RD was based on the following criteria: (1) a clinical examination showing characteristic diffuse/localized swelling or masses in single or multiple organs, (2) an elevated serum IgG4 concentration (>135 mg/dL), and (3) a histopathologic examination showing (a) marked lymphocytic and plasma cell infiltration and fibrosis or (b) infiltration of IgG4+ plasma cells (a ratio of IgG4+/ IgG + cells $>40 \%$ and $>10$ IgG4+ plasma cells per high power field). Patients with other autoimmune diseases, active infection, or malignant disease diagnosed within 5 years were excluded. The study was conducted in compliance with the Declaration of Helsinki and was approved by the Ethics Committee of Peking Union Medical College Hospital (No. S-442). All patients signed written informed consent.

\section{Clinical data and laboratory parameters}

Patients' data including age, gender, disease duration, history of allergy, treatment strategy, symptom onset, organs affected, and follow-up time were collected. Allergy history was collected using the criteria from the European Academy of Allergy and Clinical Immunology. IgG4-RD responder index (RI) (2018 version) at baseline and each follow-up was evaluated [11] Laboratory 
parameters included routine blood analysis, liver and kidney function, erythrocyte sedimentation rate (ESR), hypersensitive C-reactive protein (hsCRP); serum complement C3 and C4, serum IgG, A, and M, IgG subclass, total IgE; rheumatoid factor; and auto-antibodies tests. Affected organs and evaluation of treatment efficacy were determined by clinical symptoms, physical examinations, histopathological findings, and imaging, including ultrasonography, computed tomography (CT), magnetic resonance imaging (MRI), or positron emission tomography/computed tomography (PET/CT).

\section{Assessment of treatment outcomes}

Disease response was defined as the decline of the IgG4RD RI $\geq 2$ points compared with baseline [12]. Clinical relapse was defined as a recurrence of symptoms and signs and/or worsening of imaging studies, with or without re-elevation of the serum IgG4 level [13]. The time of relapse was defined as the date of new onset or recurrence/exacerbation of disease based on symptoms, physical examination, laboratory, or radiology findings after improvement [14].

To compare therapeutic outcomes of hypocomplementemia group and normal complement group, patients who were initially treated with initial GCs alone or GCs plus IMs, with initial GC doses of $0.5-1.0 \mathrm{mg} / \mathrm{kg} /$ day $(30-60 \mathrm{mg} /$ day $)$ of prednisone equivalent, and followed-up more than 24 months were included.

\section{Statistical analysis}

Statistical analyses were performed using the IBM SPSS Statistics version 24.0 software (IBM, Armonk, NY, USA), the Prism software version 6.1 (GraphPad Software, La Jolla, CA, USA). Data were reported as means \pm standard deviation or median and interquartile range (IQR). Normally distributed data between two groups were analyzed using independent-samples $t$ tests. Non-normally distributed data were analyzed with Mann-Whitney $U$ test. Categorical data were analyzed using the chi-square test. The correlation between serum complement level and laboratory parameters was analyzed with Pearson correlation coefficient in hypocomplementemia group at baseline. Kaplan-Meier survival curves and log-rank tests were used to compare relapse-free survival. Univariate and multivariate Cox regression analysis was performed to estimate the hazard ratio (HR) of relapse for each potential risk factor. $P$ values $<0.05$ were considered to represent significant differences between two groups.

\section{Results}

Demographic characteristics of IgG4-RD with hypocomplementemia

In this study, we prospectively enrolled 312 newly diagnosed IgG4-RD patients without treatment, 65 (20.8\%) patients had hypocomplementemia (hypocomplementemia group), 244 (78.2\%) patients had normal complement (normal complement group), and $3(1.0 \%)$ patients had elevated complement. Of the hypocomplementemia group, $45(69.2 \%)$ cases had both complement C3 and C4 reduction, $14(21.5 \%)$ cases with only C3 reduction, and $6(9.2 \%)$ cases with only $\mathrm{C} 4$ reduction. As the number of cases with elevated complement was very small, we mainly compared and discussed hypocomplementemia group and normal complement group. Demographic features of such two groups were shown in Table 1. The age at diagnose in hypocomplementemia patients was $55.85 \pm 10.89$ years, higher than normal complement group. The median duration of disease prior to initial evaluation was $12(4,36)$ months. There was no significant difference of incidence of allergic history between two groups. Compared with normal complement group, patients with hypocomplementemia showed more number of involved organs $(4.88 \pm 1.79$ vs $2.89 \pm 1.36, P<$ $0.001)$ and higher IgG4-RD RI $(15.74 \pm 5.78$ vs $9.64 \pm$ 4.33, $P<0.001)$ significantly at baseline.

\section{Comparison of involved organs in hypocomplementemia group and normal complement group}

Our data demonstrate the discrepancies in the clinical spectrums between two groups. Compared with normal complement group, patients with hypocomplementemia had significantly higher incidence of lymph node $(66.2 \%$ vs $36.1 \%, P<0.001)$, lacrimal gland $(66.2 \%$ vs $45.5 \%, P=$ $0.003)$, submandibular gland ( $63.1 \%$ vs $41.4 \%, P=0.001$ ), pancreas $(50.8 \%$ vs $27.1 \%, P<0.001)$, lung $(50.8 \%$ vs $18.0 \%, P<0.001)$, paranasal sinus $(41.5 \%$ vs $27.9 \% P=$ $0.029)$, parotid gland $(33.8 \%$ vs $11.5 \%, P<0.001)$, bile duct $(30.8 \%$ vs $14.3 \%, P=0.002)$, and prostate gland (15.4\% vs $4.1 \%, P=0.021)$ (Table 1$)$. There was no significant difference in kidney involvement between the two groups.

\section{Comparison of laboratory parameters in hypocomplementemia group and normal complement group}

The average level of serum C3 in hypocomplementemia was $0.54 \pm 0.17 \mathrm{~g} / \mathrm{L}$ (normal $0.73-1.46 \mathrm{~g} / \mathrm{L}$ ) and $\mathrm{C} 4$ was $0.061 \pm 0.047 \mathrm{~g} / \mathrm{L}$ (normal $0.10-0.40 \mathrm{~g} / \mathrm{L}$ )). We further compared the laboratory tests between two groups (Table 1) and found that patients with hypocomplementemia had significantly higher baseline levels of peripheral eosinophils count (median $0.42 \times$ $10^{9} / \mathrm{L}$ vs $\left.0.17 \times 10^{9} / \mathrm{L}, P=0.006\right), \quad$ ESR $(46.34 \pm 32.40$ $\mathrm{mm} / \mathrm{h}$ vs median $16 \mathrm{~mm} / \mathrm{h}, P<0.001), \operatorname{IgG}(30.92 \pm$ $15.31 \mathrm{~g} / \mathrm{L}$ vs $18.05 \pm 8.79 \mathrm{~g} / \mathrm{L}, P<0.001$ ), total-IgE (median $471.0 \mathrm{KU} / \mathrm{L}$ vs $222.0 \mathrm{KU} / \mathrm{L}, P<0.001), \quad$ IgG1 $(1295.11 \pm 539.48 \mathrm{mg} / \mathrm{dL}$ vs $907.73 \pm 439.79 \mathrm{mg} / \mathrm{dL}, P<$ $0.001), \quad \operatorname{IgG3}(100.56 \pm 80.81 \mathrm{mg} / \mathrm{dL}$ vs $50.54 \pm 41.23$ 
Table 1 Comparison of demographic characteristics of IgG4-RD with and without hypocomplementemia at baseline

\begin{tabular}{|c|c|c|c|}
\hline Characteristics at baseline & Hypocomplementemia Group $(n=65)$ & Normal Complement Group $(n=244)$ & $P$-value \\
\hline \multicolumn{4}{|l|}{ Demographic features } \\
\hline Gender (male), $n$ (\%) & $47(72.3 \%)$ & $144(59.0 \%)$ & 0.05 \\
\hline Age of diagnosis, mean \pm SD & $55.85 \pm 10.89$ & $53.05 \pm 13.00$ & 0.113 \\
\hline Duration of disease (medium months, IQR) & $12(4,36)$ & $12(6,48)$ & 0.131 \\
\hline Allergy history $(n, \%)$ & $40(61.5 \%)$ & $125(51.2 \%)$ & 0.139 \\
\hline Number of organs involved (mean \pm SD) & $4.88 \pm 1.79$ & $2.89 \pm 1.36$ & $<0.001^{* * *}$ \\
\hline IgG4-RD RI, mean \pm SD & $15.74 \pm 5.78$ & $9.64 \pm 4.33$ & $<0.001^{* * *}$ \\
\hline \multicolumn{4}{|l|}{ Organ involvement $(n \%)$} \\
\hline Lymph node & $43(66.2 \%)$ & $88(36.1 \%)$ & $<0.001^{* * *}$ \\
\hline Lacrimal gland & $43(66.2 \%)$ & $111(45.5 \%)$ & $0.003^{* *}$ \\
\hline Submandibular gland & $41(63.1 \%)$ & $101(41.4 \%)$ & $0.001 * *$ \\
\hline Pancreas & $33(50.8 \%)$ & $66(27.1 \%)$ & $<0.001^{* * *}$ \\
\hline Lung & $33(50.8 \%)$ & $44(18.0 \%)$ & $<0.001^{* * *}$ \\
\hline Paranasal sinus & 27 (41.5\%) & $68(27.9 \%)$ & $0.029 *$ \\
\hline Parotid gland & $22(33.8 \%)$ & $28(11.5 \%)$ & $<0.001^{* * *}$ \\
\hline Bile duct & $20(30.8 \%)$ & $35(14.3 \%)$ & $0.002^{* *}$ \\
\hline Kidney & $12(18.5 \%)$ & $26(10.7 \%)$ & 0.082 \\
\hline Prostate gland & $10(15.4 \%)$ & $10(4.1 \%)$ & $0.0218^{*}$ \\
\hline Retroperitoneum & $8(12.3 \%)$ & $46(18.9 \%)$ & 0.229 \\
\hline Aorta/artery & $5(7.7 \%)$ & $28(11.5 \%)$ & 0.442 \\
\hline Pituitary & $3(4.6 \%)$ & $7(2.9 \%)$ & 0.443 \\
\hline Gastrointestinal tract & $0(0.0 \%)$ & $4(1.6 \%)$ & \\
\hline Mediastinum & $3(4.6 \%)$ & $6(2.5 \%)$ & 0.349 \\
\hline Thyroid (Riedel's) & $3(4.6 \%)$ & $10(4.1 \%)$ & 0.835 \\
\hline \multicolumn{4}{|l|}{ Serological features } \\
\hline C3(normal 0.73-1.46 g/L) & $0.54 \pm 0.17$ & $0.99 \pm 0.33$ & $<0.001^{* * *}$ \\
\hline C4(normal 0.10-0.40 g/L) & $0.061 \pm 0.047$ & $0.19(0.14,0.25)$ & $<0.001^{* * *}$ \\
\hline $\mathrm{WBC}\left(10^{9} / \mathrm{L}\right)$ & $6.67 \pm 1.84$ & $6.54(5.58,7.75)$ & 0.894 \\
\hline $\mathrm{HGB}(\mathrm{g} / \mathrm{L})$ & $132.35 \pm 18.38$ & $134.37 \pm 22.07$ & 0.508 \\
\hline $\operatorname{PLT}\left(10^{9} / L\right)$ & $215.80 \pm 59.00$ & $240.30 \pm 75.80$ & $0.017^{*}$ \\
\hline $\operatorname{EOS}\left(10^{9} / \mathrm{L}\right)$ & $0.39(0.14,0.69)$ & $0.19(0.10,0.33)$ & $0.001 * *$ \\
\hline ESR $(\mathrm{mm} / \mathrm{H})$ & $46.34 \pm 32.40$ & $16(7,37)$ & $<0.001^{* * *}$ \\
\hline hsCRP (mg/L) (normal< 8mg/L) & $2.51(0.82,8.64)$ & $1.86(0.64,5.68)$ & 0.117 \\
\hline lgG (normal 7.0-17.0 g/L) & $30.92 \pm 15.31$ & $18.05 \pm 8.79$ & $<0.001^{* * *}$ \\
\hline IgA (normal 0.7-4.0 g/L) & $1.59 \pm 0.78$ & $2.36 \pm 1.32$ & $<0.001^{* * *}$ \\
\hline IgM (normal 0.4-2.3 g/L) & $0.87 \pm 0.63$ & $0.81(0.54,1.23)$ & 0.132 \\
\hline IgE (KU/L)(normal<60KU/L) & $471.0(246.75,880.00)$ & $222.00(63.70,609.00)$ & $<0.001^{* * *}$ \\
\hline IgG1 (normal 490-1140 mg/dL) & $1295.11 \pm 539.48$ & $907.73 \pm 439.79$ & $<0.001^{* * *}$ \\
\hline lgG2(normal 150-640 mg/dL) & $666.83 \pm 542.76$ & $612.56 \pm 256.77$ & 0.259 \\
\hline IgG3(normal 20-110 mg/dL) & $100.56 \pm 80.81$ & $50.54 \pm 41.23$ & $<0.001^{* * *}$ \\
\hline IgG4(normal 80-135 mg/dL) & $2614.13 \pm 1915.39$ & $547.50(274.23,1215.50)$ & $<0.001^{* * *}$ \\
\hline $\lg G 4 / \lg G$ & $0.80 \pm 0.44$ & $0.44 \pm 0.34$ & $<0.001^{* * *}$ \\
\hline RF positive $(n, \%)$ & $20(n=57,35.1 \%)$ & $44(n=166,26.5 \%)$ & 0.217 \\
\hline
\end{tabular}

IG4-RD RI IgG4-RD responder index, WBC white blood cell count, HGB hemoglobin, PLT platelet count, EOS eosinophil count, ESR estimated sedimentation rate, $h S C R P$ hypersensitive C-reactive protein, $I g$ immunoglobulin, $R F$-positive the level of rheumatoid factor $\geq 20 \mathrm{IU} / \mathrm{ml}$ ${ }^{*} P<0.05,{ }^{* *} P<0.01,{ }^{* * *} P<0.001$ 
$\mathrm{mg} / \mathrm{dL}, \quad P<0.001), \quad$ IgG4 $(2614.13 \pm 1915.39 \mathrm{mg} / \mathrm{dL}$ vs median $547.50 \mathrm{mg} / \mathrm{dL}, \quad P<0.001)$, ratio of $\mathrm{IgG} 4 /$ $\operatorname{IgG}(0.80 \pm 0.44$ vs $0.44 \pm 0.34, P<0.001)$, whereas significantly lower count of platelet $\left(215.80 \pm 59.00 \times 10^{9}\right.$ / $\mathrm{L}$ vs $\left.240.30 \pm 75.80 \times 10^{9} / \mathrm{L}, P=0.017\right)$ and IgA $(1.59 \pm$ $0.78 \mathrm{~g} / \mathrm{L}$ vs $2.36 \pm 1.32 \mathrm{~g} / \mathrm{L}, P<0.001)$.

\section{Correlations between serum C3, C4, and clinical characteristics at baseline}

We performed Pearson correlation coefficient analysis to investigate the association between serum complement level and age of onset, duration of disease, number of involved organs, IgG4-RD RI, and laboratory parameters including C3/C4, count of eosinophil, ESR, hsCRP, IgG,
IgA, IgM, IgE, IgG1, IgG2, IgG3, and IgG4, in total patients at baseline. As shown in Fig. 1, serums C3 and C4 showed a significant positively correlation with each other $(r=0.726, P<0.001)$. The level of serum C3 was negatively correlated with number of involved organs $(r=-0.441, P<0.001)$, IgG $(r=-0.362, P<0.001), \operatorname{IgG} 3$ $(r=-0.338, P<0.001)$, and IgG4 $(r=-0.425, P<0.001)$, whereas positively correlated with $\operatorname{IgA}(r=0.341, P<0.001)$. Similarly, serum $C 4$ level was negatively correlated with number of involved organs $(r=-0.309, P<0.001)$, laboratory parameters such as IgG $(r=-0.436, P<0.001)$, IgG1 $(r=-0.315, P<0.001)$, IgG3 $(r=-0.301, P<0.001)$, and IgG4 $(r=-0.422, P<0.001)$. In addition, serum $\mathrm{C} 3$ was weakly correlated with the age $(r=-0.162, P=0.005)$,

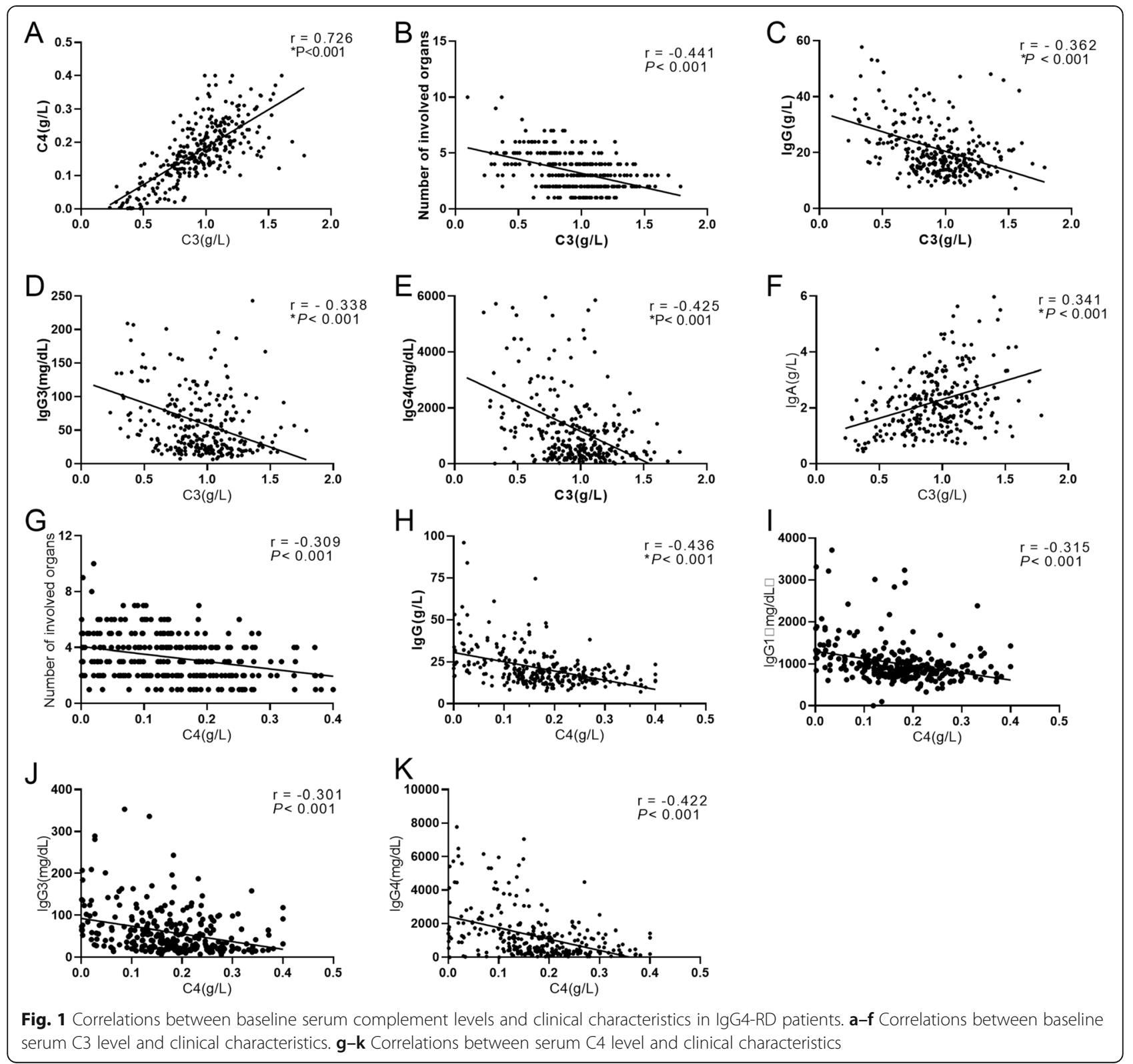



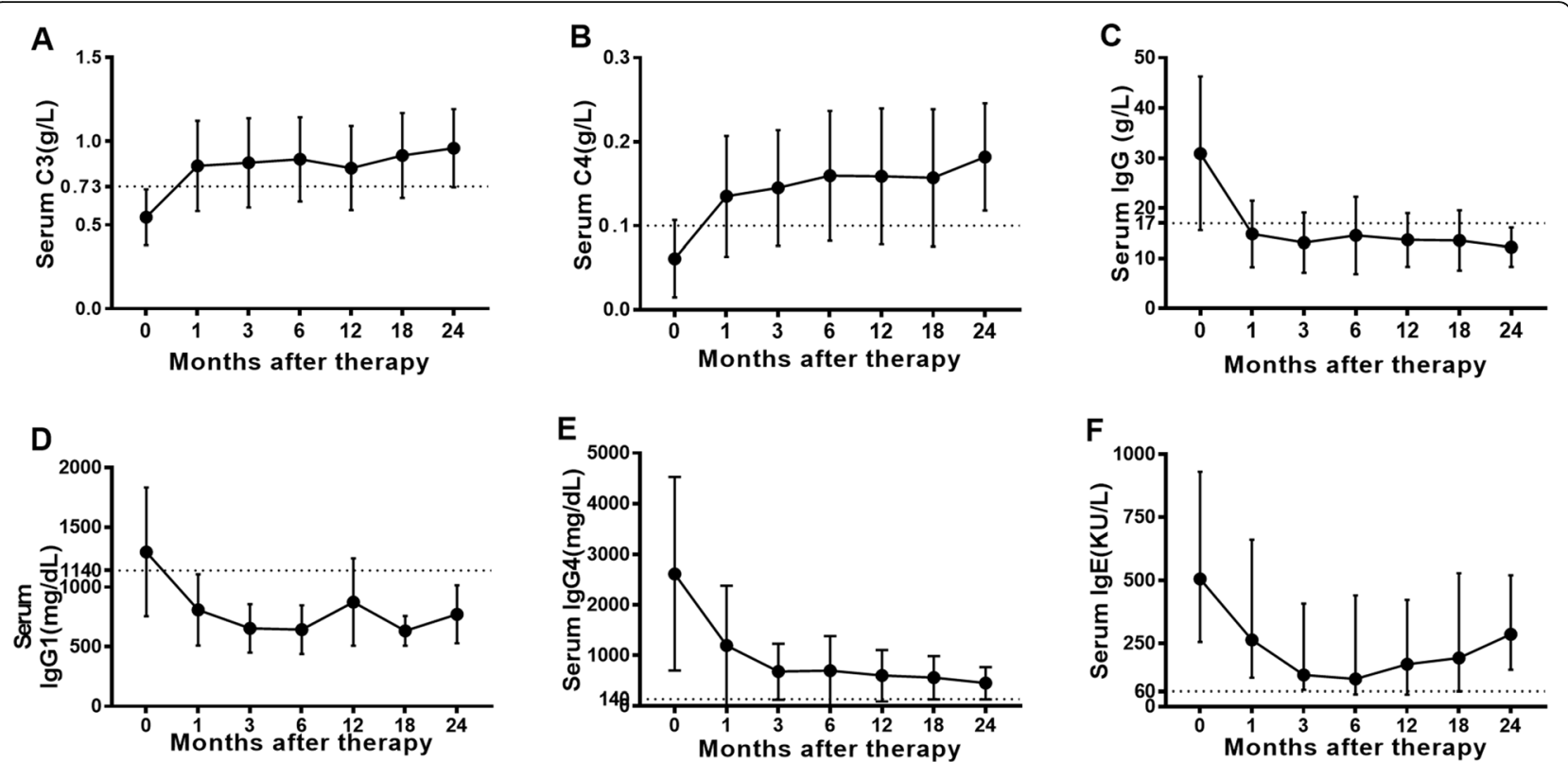

Fig. 2 Changes of serums C3 and C4, other laboratory parameters and IgG4-Rl after treatment in IgG4-RD patients with hypocomplementemia. Change of serum C3 (a), serum C4 (b), serum $\operatorname{lgG}(\mathbf{c})$, serum $\operatorname{lgG} 1(\mathbf{d})$, serum $\lg G 4(\mathbf{e})$, and serum $\lg \mathrm{E}(\mathbf{f})$ after treatment in $\lg G 4-\mathrm{RD}$ patients with hypocomplementemia

IgG4-RD RI $(r=-0.201, P=0.005)$, IgG1 $(r=-0.216, P<$ $0.001)$, and hsCRP $(r=0.203, P=0.002)$. Serum C4 was weakly correlated with IgG4-RD RI $(r=-0.207, P<0.001)$, hsCRP $(r=0.192, P=0.003)$, and IgA $(r=0.224, P<0.001)$.

Treatment efficacy in IgG4-RD with hypocomplementemia All patients with hypocomplementemia were treated with glucocorticoids (GCs), GCs combined with immunosuppressant agents (GCs plus IM) or GCs combined with rituximab (RTX). The standard induction dosage of oral prednisone was $0.5-1.0 \mathrm{mg} / \mathrm{kg} /$ day in the first month and tapered per 1 or 2 weeks to the maintenance dosage. 18 (27.7\%) patients received GC monotherapy. One (1.5\%) patient received GCs plus RTX. Others were treated with GCs plus IM, including cyclophosphamide (CYC) $(n=22,33.8 \%)$, mycophenolate mofetil (MMF) $(n=12,18.5 \%)$, methotrexate (MTX) $(n=5,7.7 \%)$, iguratimod $5(n=5,7.7 \%)$, and leflunomide $(n=1,1.5 \%)$.

The average follow-up time of IgG4-RD patients with hypocomplementemia was $34.01 \pm 18.34$ months. The level of serums $\mathrm{C} 3$ and $\mathrm{C} 4$ increased to the normal range the first month after treatment (Fig. 2a, b). Laboratory parameters such as ESR, hsCRP, IgG (Fig. 2c), IgG1 (Fig. 2d), IgG4 (Fig. 2e), and IgE (Fig. 2f) decreased significantly after treatment. Disease response occurred in 64 (98.5\%) patients at month 3 and was observed quickly. One patient had no improvement at the 3rd month until increased the dosage of GCs and combined with IMs. Fifteen $(23.1 \%)$ patients relapsed during follow-up with mean recurrence time $14.2 \pm 13.8$ months, while only $25 \%$ of them had hypocomplementemia while relapsed.

\section{Comparison of treatment and outcome in IgG4-RD hypocomplementemia group and normal complement group}

We compared the first-line treatment between the two groups. The initial doses of GCs in hypocomplementemia group were higher than the normal complement group $(37.77 \pm 14.28$ vs $31.71 \pm 18.1, \quad P=$ 0.013) (Fig. 3a). GCs-based therapies (GCs alone or in combination with IM/RTX) were used more frequently in hypocomplementemia group $(100 \%$ vs $85.7 \%, P=0.001)$. Higher IgG4-RI and more organs involved in hypocomplementemia group, indicating that the different treatment regimens between the two groups.

To compare therapeutic outcomes of hypocomplementemia group and normal complement group, 38 patients with hypocomplementemia and 105 patients with normal complement who were initially treated with initial GC alone or in combination with IMs, with initial GC doses of $0.5-1.0 \mathrm{mg} / \mathrm{kg} /$ day $(30-60$ $\mathrm{mg} /$ day) of prednisone equivalent, and followed-up more than 24 months were included. The results showed that although IgG4-RD RI score was higher in hypocomplementemia group at baseline and 6th month follow-up $(P<0.001$ and $P=0.005$, respectively), there was no significant difference between two groups at 12, 24, 36, and 48 months after therapy 


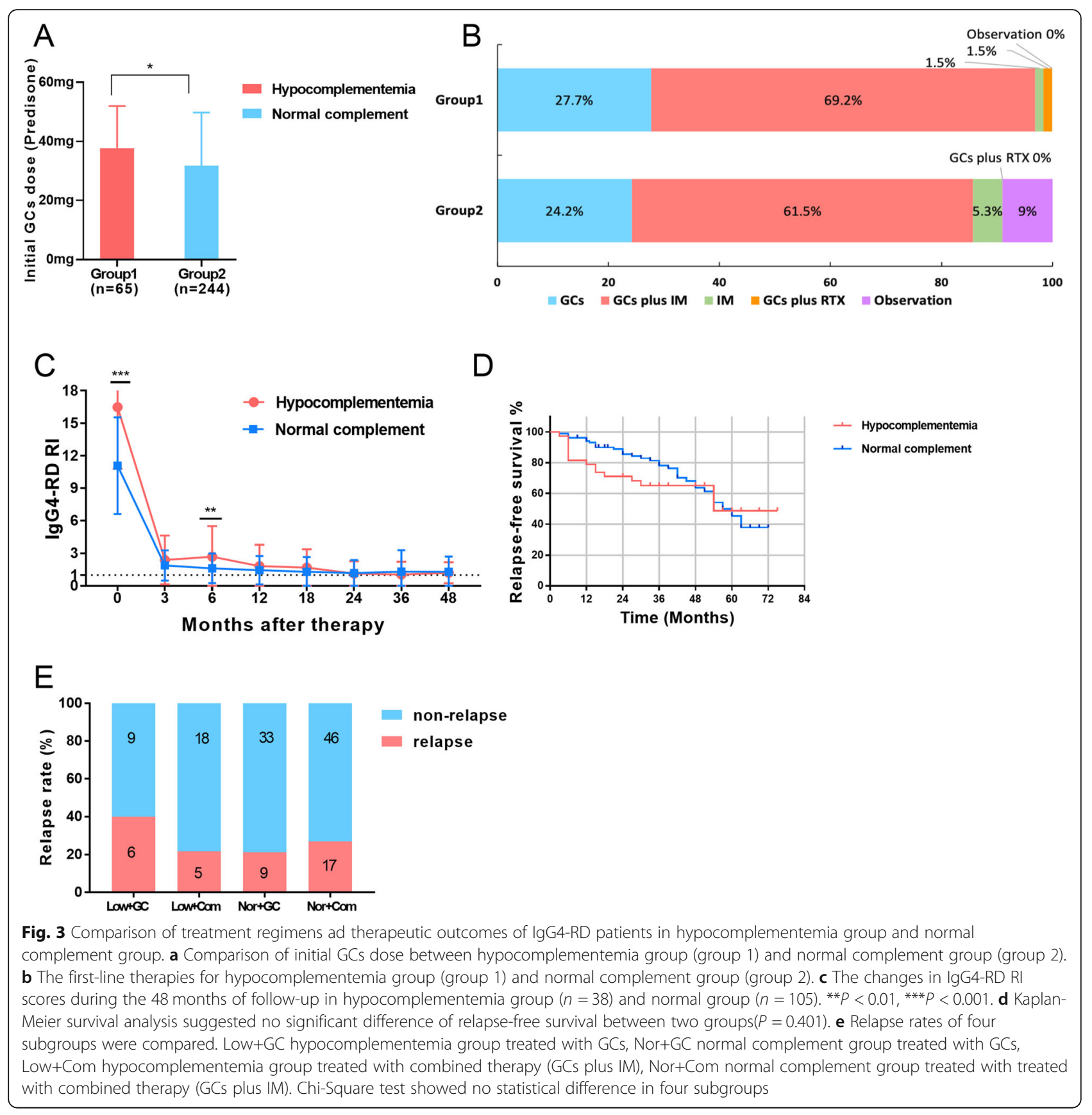

(Fig. 3c). Relapse rate was observed in in $21.9 \%$, $29.0 \%, 29.0 \%$, $35.0 \%$, and $51.2 \%$ of the patients with hypocomplementemia at $12,24,36,48$, and 60 months after therapy, respectively. Kaplan-Meier survival analysis suggested no significant difference of relapse-free survival between two groups $(P=0.401)$ (Fig. 3d). Relapse rates were analyzed in four subgroups after adjustment to hypocomplementemia patients treated with GC monotherapy group $(n=15)$, hypocomplementemia patients treated with combined therapy group $(n=23)$, normal complement GCs treated with monotherapy group $(n=42)$, and normal complement treated with combined therapy group $(n=63)$. The relapse rates of four subgroups within 24 months were $40.0 \%, 21.7 \%, 21.4 \%$, and $27 \%$, respectively. The relapse rate of hypocomplementemia GC monotherapy group was higher than hypocomplementemia combination therapy group $(P=0.225)$ without statistical difference, as well as normal complement GC monotherapy group ( $P=0.161)$ (Fig.3e). 


\section{Discussion}

As far as we know, this is the first study to elaborate the clinical and laboratory characteristics, treatment response, and prognosis of IgG4-RD with hypocomplementemia. Further, we investigated serum C3 and C4 would be biomarkers for disease activity.

In our prospective cohort, complements C3 and/or C4 were decreased in one fifth of the IgG4-RD patients. Compared with normal complement group, IgG4-RD with hypocomplementemia was a distinct clinical phenotype, which with higher number of involved organs, higher disease activity, and disparities of involved organs. John Stone suggested that IgG4-RD consisted of two overlapping subsets: a proliferative type and fibrotic type. Patients with the proliferative subset of IgG4-RD tend to have disease affecting the glandular and epithelial tissues and have high serum concentrations of IgG4, IgG1, and IgE; a higher likelihood of hypocomplementemia [15]. Our study demonstrated that IgG4-RD with hypocomplementemia had higher incidence of lymphadenopathy, dacryoadenitis, sialadenitis, autoimmune pancreatitis, lung disease, paranasal sinusitis, sclerosing cholangitis, and prostate gland involvement, compared with normal complement group. Parameters associated with inflammatory and high disease activity include count of eosinophil, ESR, IgG, IgE, IgG1, IgG3, and IgG4 were significantly higher. Therefore, we think that hypocomplementemia is one of the most important features of proliferative subset.

As we known, hypocomplementemia is one important inclusion criteria associated with kidney involvement in 2019 American College of Rheumatology/European League Against Rheumatism classification criteria for IgG4-RD [16]. Kawano reported more than $50 \%$ of patients with active IgG4-tubulointerstitial nephritis (TIN) which is the most common manifestation of IgG4-RD with kidney involvement had hypocomplementemia [17]. However, in our cohort, there was no significant difference in renal involvement between hypocomplementemia and normal complement groups. Since apart from IgG4-TIN, IgG4-related glomerular nephritis, renal parenchymal nodule lesions, and renal pelvis involvement were considered as renal involvement in our study. As Teng et al. studied 65 IgG4-related urinary disease (RUD) patients, TIN only accounted for 21 (32.3\%) of IgG4-RUD and the mean serum C3 level of TIN group was significantly lower than other groups. The mean serums C3 and C4 were normal in a group of renal pelvis or ureter involvement, abnormal renal radiological findings, and renal parenchymal lesions accompanied by retroperitoneal fibrosis [18].

We compared treatment outcomes between two groups. After 1-month treatment, the average level of serums $\mathrm{C} 3$ and $\mathrm{C} 4$ in hypocomplementemia group recovered swiftly. Hypocomplementemia at baseline would not be the predictor for prognosis as there was no significant difference of relapse rate within 72 months in two groups after treatment. However, considering that patients with hypocomplementemia were given more aggressive treatment because of the higher disease activity, the predictive value of hypocomplementemia for prognosis of IgG4-RD in this study may be biased. We compared relapse rates in four subgroups after matching treatment. The relapse rate of hypocomplementemia with GC monotherapy group was $40.0 \%$ higher than other groups, while there was no statistics difference possible due to the small sample size. Prospective cohort study with larger sample size is needed.

Mechanism of complement activation in IgG4-RD remains not clear. It was reported that anti-galectin-3 [19], anti-annexin A11 [20], anti-laminin-511 [21], and anti-prohibitin [22] had been detected in a minority of patients with IgG4-RD, for example, antigalectin-3 antibodies were identified in approximately $30 \%$ of a cohort of 121 IgG4-RD patients with multiple organ involvement [19]. Therefore, antigenantibody immune complex may play a role in activating complement pathway. Muraki et al. proposed that based on the high serum circulating immune complex in autoimmune pancreatitis, the classical complement activation pathway is thought to be involved in IgG4RD [23]. As we all known, IgG4 molecule does not bind complement effectively and is unable to activate complement pathway, and one plausible explanation is elevated IgG1 played a prominent role via the classical complement pathway $[1,24]$. As we also found IgG1 elevated remarkably in hypocomplementemia group and serums C3 and C4 were negatively correlated with IgG1. After treatment, IgG1 decreased along with the increase of serums $\mathrm{C} 3$ and $\mathrm{C} 4$ to normal in the first month, whereas the decline of IgG4 lagged behind. Another potential explanation is that IgG4 may activate the complement system through the MBL pathway [24, 25]. Sugimoto et al. reported that high serum levels of C1q-binding IgG4 in IgG4RD patients with hypocomplementemia. They observed marked reduction of total complement hemolytic (CH50) and complement activity in the classical complement pathway as well as the MBL pathway in normal human serum incubated with polyethylene glycol precipitates-immune complexes isolated from IgG4RD patients with hypocomplementemia [25]. Altered glycosylation of IgG1 and IgG4 antibody subclasses might also have a role in causing hypocomplementemia in patients with IgG4-RD [26]. Interestingly, IgA in hypocomplementemia group was significantly lower than normal complement group and positively correlated with serum C3 and C4 level. 
The role of IgA in the complement activation pathway remains a mystery.

\section{Conclusion}

In conclusion, IgG4-RD patients with hypocomplementemia compared to normal complement patients had higher disease activity, higher number of affected organs and proliferative subtype features. The levels of serum C3 and C4 were negatively correlated with indicators of disease activity number of involved organs and laboratory parameters such as IgG, IgG3, and IgG4, and implicated serums $\mathrm{C} 3$ and $\mathrm{C} 4$ could be the biomarkers for disease activity. The low level of serums C3 and C4 could be recovered quickly after immunosuppressive therapy. IgG4-RD patients with hypocomplementemia respond well to treatment and have no significant difference of relapse rate in IgG4-RD patients with normal complement.

\section{Abbreviations \\ IgG4-RD: Immunoglobulin G4-related disease; RI: Responder index; IgG: Immunoglobulin G; hsCRP: Hypersensitive C-reactive protein; MBL: Mannan-binding lectin; SLE: Systemic lupus erythematosus; APS: Antiphospholipid syndrome; AAV: Anti-neutrophil cytoplasmic antibody associated vasculitis; CT: Computed tomography; MRI: Magnetic resonance imaging; PET/CT: Positron emission tomography/computed tomography; GCs: Glucocorticoids; IM: Immunosuppressant; RTX: Rituximab; CYC: Cyclophosphamide; MMF: Mycophenolate mofetil; MTX: Methotrexate; IQR: Interquartile range; HR: Hazard ratio; TIN: Tubulointerstitial nephritis; RUD: Urinary disease}

\section{Acknowledgements}

Not applicable.

\section{Authors' contributions}

LYP and $H L$ designed the research, analyzed the data, and wrote the manuscript. JXZ, PPZ, JQL, ZL, DW, SZZ, YJY, WB, and LW helped with the diagnosis, data collection, and follow-up. YYF helped with the data analysis. $Y Z, X F Z, F C Z$, and WZ designed the study and revised the manuscript. The authors read and approved the final manuscript.

\section{Funding}

This work was supported by the CAMS Initiative for Innovative Medicine [2017-I2M-3-001] and the National Natural Science Foundation of China [81771757, 81601433], the Capital Health Research and Development Special (2020-2-4017), the Non-profit Central Research Institute Fund of Chinese Academy of Medical Sciences (NWB20203346), and the National Key Research and Development Program of China" (No. 2016YFC0901500)

\section{Availability of data and materials}

The datasets used and/or analyzed during the current study are available from the corresponding author on reasonable request.

\section{Declarations}

\section{Ethics approval and consent to participate}

The study was approved by the Ethics Committee of Peking Union Medical College Hospital and approval number is S-442. All enrolled patients consented to attend this cohort study and signed written.

\section{Consent for publication}

Yes, we obtained consent for publication from all the individuals of whom detailed information was included in the manuscript.

\section{Competing interests}

The authors declare that they have no competing interests.

Received: 17 January 2021 Accepted: 15 March 2021

Published online: 07 April 2021

\section{References}

1. Wallace ZS, Deshpande V, Mattoo H, Mahajan VS, Kulikova M, Pillai S, Stone $J$ H. IgG4-related disease: clinical and laboratory features in one hundred twenty-five patients. Arthritis Rheumatol. 2015;67(9):2466-75. https://doi. org/10.1002/art.39205.

2. Kamisawa T, Zen Y, Pillai S, Stone JH. IgG4-related disease. Lancet. 2015; 385(9976):1460-71. https://doi.org/10.1016/S0140-6736(14)60720-0.

3. Bryan AR, Wu EY. Complement deficiencies in systemic lupus erythematosus. Curr Allergy Asthma Rep. 2014;14(7):448. https://doi.org/10.1 007/s11882-014-0448-2.

4. Holers VM. The complement system as a therapeutic target in autoimmunity. Clin Immunol. 2003;107(3):140-51. https://doi.org/10.1016/S1 521-6616(03)00034-2.

5. Vignesh $P$, Rawat $A$, Sharma M, Singh S. Complement in autoimmune diseases. Clin Chim Acta. 2017;465:123-30. https://doi.org/10.1016/j.cca.201 6.12.017.

6. Macedo AC, Isaac L. Systemic lupus erythematosus and deficiencies of early components of the complement classical pathway. Front Immunol. 2016; 24(7):55.

7. Vasilev W, Noe R, Dragon-Durey MA, Chauvet S, Lazarov VJ, Deliyska BP, Fremeaux-Bacchi V, Dimitrov JD, Roumenina LT. Functional characterization of autoantibodies against complement component C3 in patients with lupus nephritis. J Biol Chem. 2015;290(42):25343-55. https://doi.org/10.1074/ jbc.M115.647008.

8. Chen M, Jayne DRW, Zhao MH. Complement in ANCA-associated vasculitis: mechanisms and implications for management. Nat Rev Nephrol. 2017; 13(6):359-67. https://doi.org/10.1038/nrneph.2017.37.

9. Umehara $H$, Okazaki K, Masaki Y, Kawano M, Yamamoto M, Saeki T, Matsui S, Yoshino T, Nakamura S, Kawa S, Hamano H, Kamisawa T, Shimosegawa T, Shimatsu A, Nakamura S, Ito T, Notohara K, Sumida T, Tanaka Y, Mimori T, Chiba T, Mishima M, Hibi T, Tsubouchi H, Inui K, Ohara H. Comprehensive diagnostic criteria for lgG4-related disease (IgG4-RD), 2011. Mod Rheumatol. 2012;22(1):21-30. https://doi.org/10.31 09/s10165-011-0571-z

10. Umehara H, Okazaki K, Nakamura T, Satoh-Nakamura T, Nakajima A, Kawano M, Mimori T, Chiba T. Current approach to the diagnosis of IgG4-related disease - combination of comprehensive diagnostic and organ-specific criteria. Mod Rheumatol. 2017;27(3):381-91. https://doi.org/10.1080/143 97595.2017.1290911.

11. Wallace ZS, Khosroshahi A, Carruthers MD, Perugino CA, Choi H, Campochiaro C, Culver EL, Cortazar F, Della-torre E, Ebbo M, Fernandes A, Frulloni L, Hart PA, Karadag O, Kawa S, Kawano M, Kim MH, Lanzillotta M, Matsui S, Okazaki K, Ryu JH, Saeki T, Schleinitz N, Tanasa P, Umehara H, Webster G, Zhang W, Stone JH. An international multispecialty validation study of the lgG4-related disease responder index. Arthritis Care Res. 2018;70(11):1671-8. https:/doi.org/10.1 002/acr.23543.

12. Carruthers MN, Topazian MD, Khosroshahi A, Witzig TE, Wallace ZS, Hart PA, Deshpande V, Smyrk TC, Chari S, Stone JH. Rituximab for lgG4-related disease: a prospective, open-label trial. Ann Rheum Dis. 2015;74(6):1171-7. https://doi.org/10.1136/annrheumdis-2014-206605.

13. Shirakashi M, Yoshifuji H, Kodama Y, Chiba T, Yamamoto M, Takahashi H, Uchida K, Okazaki K, Ito T, Kawa S, Yamada K, Kawano M, Hirata S, Tanaka Y, Moriyama M, Nakamura S, Kamisawa T, Matsui S, Tsuboi H, Sumida T, Shibata M, Goto H, Sato Y, Yoshino T, Mimori T. Factors in glucocorticoid regimens associated with treatment response and relapses of IgG4-related disease: a multicentre study. Sci Rep. 2018;8(1):10262. https://doi.org/10.103 8/s41598-018-28405-x

14. Yunyun F, Yu P, Panpan Z, et al. Efficacy and safety of low dose Mycophenolate mofetil treatment for immunoglobulin G4-related disease: a randomized clinical trial. Rheumatology (Oxford, England). 2018;58:52-60.

15. Zhang W, Stone JH. Management of IgG4-related disease. Lancet Rheumatol. 2019;1(1):e55-65. https://doi.org/10.1016/S2665-9913(19)3 0017-7. 
16. Wallace ZS, Naden RP, Chari S, Choi HK, Della-Torre E, Dicaire JF, Hart PA, Inoue D, Kawano M, Khosroshahi A, Lanzillotta M, Okazaki K, Perugino CA, Sharma A, Saeki T, Schleinitz N, Takahashi N, Umehara H, Zen $\mathrm{Y}$, Stone JH, Members of the ACR/EULAR IgG4-RD Classification Criteria Working Group. The 2019 American College of Rheumatology/ European league against rheumatism classification criteria for lgG4related disease. Ann Rheum Dis. 2020;79(1):77-87. https://doi.org/10.113 6/annrheumdis-2019-216561.

17. Kawano M, Saeki T, Nakashima H, Nishi S, Yamaguchi Y, Hisano S, Yamanaka N, Inoue D, Yamamoto M, Takahashi H, Nomura H, Taguchi T, Umehara H, Makino H, Saito T. Proposal for diagnostic criteria or lgG4-related kidney disease. Clin Exp Nephrol. 2011;15(5):615-26. https://doi.org/10.1007/s10157011-0521-2.

18. Teng $F$, Lu H, Zhen K, et al. Urinary system manifestation of IgG4related disease: clinical, laboratory, radiological, and pathological spectra of a Chinese single- centre study. J Immunol Res. 2020;2020: 5851842.

19. Perugino CA, AISalem SB, Mattoo $H$, et al. Identification of galectin-3 as an autoantigen in patients with IgG4-related disease. J Allergy Clin Immunol. 2019;143(2):736-745.e6.

20. Hubers LM, Vos $H$, Schuurman AR, Erken R, Oude Elferink RP, Burgering $B$, van de Graaf S, Beuers U. Annexin A11 is targeted by lgG4 and lgG1 autoantibodies in IgG4-related disease. Gut. 2018;67(4):728-35. https://doi. org/10.1136/gutjnl-2017-314548.

21. Shiokawa M, Kodama Y, Sekiguchi K, et al. Laminin 511 is a target antigen in autoimmune pancreatitis. Sci Transl Med. 2018:10(453):eaaq0997.

22. Du H, Shi L, Chen P, et al. Prohibitin is involved in patients with lgG4 related disease. PLoS One. 2015;10(5):e0125331. https://doi.org/10.1371/ journal.pone.0125331.

23. Muraki T, Hamano H, Ochi Y, Komatsu K, Komiyama Y, Arakura N, Yoshizawa K, Ota M, Kawa S, Kiyosawa K. Autoimmune pancreatitis and complement activation system. Pancreas. 2006;32(1):16-21. https://doi.org/10.1097/01. mpa.0000188308.75043.e4

24. Kawa S. The immunobiology of immunoglobulin $\mathrm{G} 4$ and complement activation pathways in IgG4-related disease. Curr Top Microbiol Immunol. 2017:401:61-73. https://doi.org/10.1007/82_2016_39.

25. Sugimoto M, Watanabe $H$, Asano $T$, Sato $\mathrm{S}$, Takagi T, Kobayashi $\mathrm{H}$, Ohira $\mathrm{H}$. Possible participation of lgG4 in the activation of complement in lgG4related disease with hypocomplementemia. Mod Rheumatol. 2016;26(2): 251-8. https://doi.org/10.3109/14397595.2015.1076924

26. Culver EL, van de Bovenkamp FS, Derksen NIL, Koers J, Cargill T, Barnes E, de Neef LA, Koeleman CAM, Aalberse RC, Wuhrer M, Rispens T. Unique patterns of glycosylation in immunoglobulin subclass G4-related disease and primary sclerosing cholangitis. J Gastroenterol Hepatol. 2019;34(10):1878-86. https:// doi.org/10.1111/jgh.14512.

\section{Publisher's Note}

Springer Nature remains neutral with regard to jurisdictional claims in published maps and institutional affiliations.

Ready to submit your research? Choose BMC and benefit from:

- fast, convenient online submission

- thorough peer review by experienced researchers in your field

- rapid publication on acceptance

- support for research data, including large and complex data types

- gold Open Access which fosters wider collaboration and increased citations

- maximum visibility for your research: over $100 \mathrm{M}$ website views per year

At $\mathrm{BMC}$, research is always in progress.

Learn more biomedcentral.com/submissions 